\title{
Differences in approach run kinematics: successful vs. unsuccessful jumps in the pole vault.
}

J. Cassirame, H. Sanchez, T. A. Exell, V. Panoutsakopoulos, A. S. Theodorou, S. Homo \& J. Frere

\begin{abstract}
5

This study investigated biomechanical differences between successful and unsuccessful jumps during a pole vault competition. Two hundred and seven pairs of successful and unsuccessful jumps at the same height were analysed. Participants included male and female athletes of three different age groups with bar height clearances ranging from 2.81 to $5.91 \mathrm{~m}$. Run-up parameters were collected using an Optojump Next system and a Stalker Pro II radar gun. A 2D kinematical analysis was conducted to obtain selected parameters of the take-off. Only trivial and small differences were found between successful and unsuccessful jumps. Speed at last touchdown showed a significant small difference between successful and unsuccessful jumps, as greater speed at take-off $(+0.15 \mathrm{~m} / \mathrm{s})$ was observed at successful jumps compared to unsuccessful jumps. Furthermore, female athletes showed a significant small difference in horizontal hand-foot distance between successful jumps and unsuccessful jumps $(+0.05 \mathrm{~m}$ and $+0.06 \mathrm{~m}$ at pole plant and take-off, respectively). The results suggest that pole vaulters should produce a fast run-up and avoid a decrease in speed before take-off. Small adjustments in the takeoff posture might increase the transfer of energy from the athlete to the pole and thus an improvement concerning the height of bar clearance.
\end{abstract}

Key-words: Performance, Step parameters, Biomechanics, Analysis, Asymmetry 


\section{Introduction}

Pole vaulting is a complex athletics discipline that requires athletes to posses many different qualities in order to achieve high performance. Vaulters have to combine a high technical ability with many physical capabilities such as speed, strength and agility or gymnastic capabilities. This requirement is due to the different tasks involved in the pole vault technique, as its main temporal phases are the (1) run-up, (2) pole planting and take off, (3) pole bending and straightening, and (4) bar clearance (Frère, L'hermette, Slawinski, \& Tourny-Chollet, 2010).

Pole vaulting has been largely analyzed and described during international competitions to obtain better understanding of maximal performance (Angulo-Kinzler et al., 1993; Schade, Arampatzis, Brüggemann, \& Komi, 2004; Zagorac, 2013). The advantage of conducting analyses during official competitions is the increased ecological validity, maximal intensity of performers and a better understanding of run-up parameters that limit performance (Christensen \& Zebas, 2000). Biomechanical analyses of pole vault performance have highlighted key performance determinants which provide reference to coaches that can guide their training program or be used by athletics federations to build their program (Christensen, Francis, Keller, Strand, \& Hatterman-Valenti, 2014; Decker \& Bird, 2004; Vaslin \& Cid, 1993). Based on the recommendations from these sources, coaches focus on parameters such as approach speed, grip length or take-off distance among others.

Research on pole-vault run-up parameters has shown that athletes tend to accelerate until the instant of take-off (Makaruk, Porter, Starzak, \& Szymczak, 2016). Athletes also need to make small adjustments to their movement patterns and thus they regulate their locomotion in response to visual or physical feedback in order to achieve a consistent take-off point (Needham, Exell, Bezodis, \& Irwin, 2018). This run-up variability can be dysfunctional and lead to unsuccessful jumps, or functional and serve as a compensatory response to changes in environmental factors or other biomechanical variables during the attempt, thus producing a consistent performance outcome (Theodorou, Panoutsakopoulos, Exell, et al., 2017).

Past research has shown that pole vaulters also use different movement patterns during performances at different heights of the bar (Starzak, Makaruk, \& Niznikowski, 2016). For example, at their best successful jump, pole-vaulters increased their approach velocity by increasing step frequency to a greater extent than step length (Theodorou, Panoutsakopoulos, \& Exell, 2016). However, it was found that when attempting to clear a greater height than their best successful jump, pole-vaulters were less reliant on step frequency to increase step velocity (Theodorou, Panoutsakopoulos, Exell, \& Vujkov, 2017). Currently, it is unclear if such contradictory results arise from differences due to age and the level of expertise of the athletes, or arise from differences in the task constraint. Whilst functional variability may be desirable, any slight deviation in technique that occurs during a jump could be irreversible and lead to an unsuccessful jump if other factors remain similar. Regarding dysfunctional variability, it has been observed that pole vaulters demonstrated a more variable gait regulation strategy in unsuccessful jumps compared to successful jumps (Tamura, Nunome, \& Usui, 2017). Thus, it is important to investigate the step parameter patterns and the 
biomechanics of the take-off between successful and unsuccessful jumps at the same height, and to consider the possible differences across gender, age, and level of expertise.

The aim of this study was to investigate the kinematic differences in the pole vault run-up between successful and unsuccessful jumps during an indoor competition. We hypothesized that unsuccessful jumps would be associated with associated with a slower approach velocity, an irregular pattern of step parameter progression, and less favorable take-off parameters. The purpose of the study was to inform coaches and enhance feedback to athletes with information to be used between competitive jumps and in training, by identifying the most relevant parameters that determine a successful bar clearance.

\section{Methods}

\section{Experimental design}

Data were collected between 2015 and 2017 at the following competitions: the National Elite French Championships, the National French Youth Championships, and the 2016 and 2017 All-Star Perche International Meeting. All measurements were performed in indoor facility to avoid possible environmental effects (i.e. wind). Data acquisition during athletics events was selected as it is well established that, during an official competition, athletes perform at maximal intensity and thus they exhibit more representational to their abilities values for the run-up parameters (Christensen, 2004). The experimental set-up was the same in all competitions. The setting did not interfere with the athletes and therefore it did not affect their performance.

\section{Jump selection}

Jumps included in the analysis were always performed in the same competition. Successful (SU) and unsuccessful (UN) jumps were analyzed for athletes that failed to clear a height and then cleared the same height at a subsequent jump. In this pair of jumps, the athletes always used the same run-up distance and the same pole. This approach eliminates the potential interfering effects of the track surface (Cassirame, Sanchez, \& Morin, 2017), the athlete's approach speed (Linthorne \& Weetman, 2012) and the properties of the pole (Warburton, James, Lyttle, \& Alderson, 2016). Attempts in which the athletes did not take-off and ran through were excluded. Using the above criteria, 207 pairs of unsuccessful and successful jumps were selected from the database for further analysis. The bar clearance height of the selected jumps ranged from 2.81 to $5.91 \mathrm{~m}$.

\section{Participants}

Data were collected from 132 pole-vaulters from six different categories: cadet women $(\mathrm{CW}$; $\mathrm{n}=19)$, cadet men $(\mathrm{CM} ; \mathrm{n}=25)$, junior women (JW; $\mathrm{n}=20)$, junior men $(J M ; n=20)$, elite women $(E W ; n=26)$ and elite men $(E M ; n=22)$. The cadet groups included athletes from 16 to 17 years old, the junior groups included athletes from 18 to 19 years old, and elite athletes were 20 years old or older at the time of data collection. All athletes were free from injury when data were collected. All athletes were informed about the measurements during 
competitions and provided signed consent to participate. This study was conducted in accordance with the recommendations of the Declaration of Helsinki.

\section{Data Acquisition and analysis}

Twenty meters of the Optojump Next (Microgate, Bolzano, Italy) optoelectronic system was installed on each side of the official runway to measure run-up kinematics (Ammann, Taube, \& Wyss, 2015). Due to the landing mat, the Optojump Next system could not be placed all the way up to the pole planting box and was installed up until 2.00 or $2.20 \mathrm{~m}$ before the plant box, depending on the mat configuration. To obtain the exact position of the feet during the run-up, the horizontal distance between the beginning of the Optojump Next system and the bottom of the planting box was measured, as shown in Figure 1. The step parameters were calculated for the $3^{\text {rd }}$ up to $8^{\text {th }}$ last step of the approach. The last two steps of the approach were excluded from the analysis as they are commonly used by pole-vaulters to adjust take-off distance and are not representative of run-up as previously observed by Makaruk et al. (2016). This configuration allowed direct measurement of contact time on the floor $\left(t_{c}\right)$, aerial time when the athlete was airborne $\left(\mathrm{t}_{\mathrm{a}}\right)$, step rate (SR) and step length (SL), using the spatiotemporal parameters provided by the Optojump Next Software. SL asymmetry ( $\mathrm{SL}_{\text {asy }}$ ) was calculated as the absolute difference of the horizontal displacement covered on three left-foot steps (from left foot touchdown to subsequent right foot touchdown) minus the horizontal distance covered on three right-foot steps (from right foot touchdown to subsequent left foot touchdown). SL variability ( $\mathrm{SL}_{\mathrm{var}}$ ) was calculated as the mean of the differences between step lengths over successive steps using data from the same 6 steps used to calculate SLasy. Finally, last step adjustment $\left(\mathrm{S}_{\text {adj }}\right)$ was calculated by subtracting the final SL from the penultimate SL. A negative $\mathrm{S}_{\text {adj }}$ indicated a reduction in the last SL and a positive value indicated a longer final step. The horizontal distance of the support foot toes at take-off (PoTk) and at six steps before take-off (Po6S) from the end of the planting box was calculated using the spatial data measured by the Optojump Next system (Figure 1).

The speed of the entire approach run was measured using a radar gun (Stalker Pro II, Applied Concepts, Inc., Plano, TX) positioned behind the landing mat in the run-up direction at a 1.4 $\mathrm{m}$ height to allow direct sight of athletes' torsos along the approach runway (Figure 1). The radar provided horizontal running speed at a sampling rate of $46.9 \mathrm{~Hz}$. Data from the radar gun were integrated into the MookyStalker software (Matsport, Saint-Ismier, France) and synchronized with the data from the Optojump Next system. Average speed over selected sections of the approach run was calculated after the application of a median filter on the acquired data. This method is commonly used in track and field research (Cassirame et al., 2017). Average speed was calculated for the following sections: 20 to $15 \mathrm{~m}$ (Sp1), 15 to $10 \mathrm{~m}$ (Sp2), and 10 to $5 \mathrm{~m}$ (Sp3) from the end of the planting box. Speed at last touchdown (SpTK) was considered as the average recorded the period $0.2 \mathrm{~s}$ before the instant of the last contact. From those measures, the progression of speed was calculated as $\Delta 1=\mathrm{Sp} 2-\mathrm{Sp} 1, \Delta 2=\mathrm{Sp} 3-\mathrm{Sp} 2$ and $\Delta 3=$ SpTk-Sp3.

In addition, video images of the take-off were collected at a frequency of 200 fps. A xiQUSB3 camera (xImea Gmhb, Muster, Germany) was positioned at a distance of $4 \mathrm{~m}$ 
perpendicular to the run-up and at a $3.5 \mathrm{~m}$ distance from the planting box along the direction of the runway. This setting allowed the recording of the take-off in the middle of the field of view and to reduce possible parallax error. Before each competition, calibration images were collected using a calibration pole of known length $(2.00 \mathrm{~m})$ in the athletes' sagittal plane of motion to allow distance measurements. Video images were manually processed with the Kinovea 08.15 software (Joan Charmant \& Contributors, Bordeaux, France) to extract spatial measurements at two key instants, the pole plant and the take-off. The first position occurred when the athlete was in contact with the ground at the instant of pole plant (i.e., the first video frame which showed the grip/upper hand being pushed backward). The second position occurred at the instant where the athlete took off from the ground, as defined by the Optojump Next software (Figure 2). The height of the grip hand from the ground was measured and noted as $\mathrm{H} 1$ and $\mathrm{H} 2$ for the pole plant and the take-off, respectively. In addition, the horizontal distance between the grip hand and the take-off foot's toes was calculated at the two instants and noted as U1 and U2. If the hand was posterior to the toe, the value was negative. $\Delta \mathrm{H}$ and $\Delta \mathrm{U}$ were calculated as $\Delta \mathrm{H}=\mathrm{H} 2-\mathrm{H} 1$ and $\Delta \mathrm{U}=\mathrm{U} 2-\mathrm{U} 1$ in order to obtain the vertical and horizontal displacement of the grip hand between the two instants.

\section{Statistical analysis}

Statistical analysis was performed using the Sigmaplot v12 software (SAX Software, Karlsruhe, Germany) and Microsoft Excel 2007 (Microsoft, Redmond, USA). At first, withingroups Mean and Standard Deviation (SD) were calculated for all parameters along with differences between both conditions (successful vs. unsuccessful jump). Secondly, all data were log-transformed to reduce bias arising from non-uniformity error. Differences between successful and unsuccessful jumps were expressed with standardized differences or effect size with 90\% confidence intervals (Hopkins, Marshall, Batterham, \& Hanin, 2009). Limit probabilities were also calculated to establish whether the true changes/differences were lower than, similar to, or higher than the smallest worthwhile changes/differences $(0.2 \times$ betweensubjects SD). Changes were categorized as 0-0.2 (Trivial), 0.2-0.6 (Small), 0.6-1.2 (Moderate) and 1.2-2.0 (Large; Hopkins et al., 2009). This method was applied to compare between unsuccessful and successful jumps for each group separately.

\section{Results}

Results from all measurements for each group are presented in Table 1, 2 and 3. Results of the statistical analyses are presented in Figure 3 for women and Figure 4 for men. All differences between successful and unsuccessful jumps were trivial or small. Each group presented individual combinations of small differences between successful and unsuccessful jumps. However, all groups showed a small increase in take-off speed at the successful compared to the unsuccessful jumps. Differences in take-off speed between successful and unsuccessful conditions ranged from $0.08 \mathrm{~ms}^{-1}$ for elite women to $0.18 \mathrm{~ms}^{-1}$ for cadet women. All female groups demonstrated small differences between successful and unsuccessful jumps for the hand-foot horizontal at both the instants of the pole plant and the take-off with larger values observed at the successful jumps (pole plant: $32.9-37.7 \mathrm{~cm}$, take-off: $14.9-18.5 \mathrm{~cm}$ ) than the unsuccessful jumps (pole plant: 27.6 - $30.9 \mathrm{~cm}$, take-off: $10.8-13.4 \mathrm{~cm}$ ). Position at 
take-off showed small differences between successful and unsuccessful jumps for junior women, elite women and junior males $(0.09,0.07$ and $0.06 \mathrm{~m}$, respectively), with larger values recorded for the unsuccessful jumps.

\section{Discussion}

The aim of this study was to investigate biomechanical differences between successful and unsuccessful jumps during indoor pole vault competition. Results of the study covered a large cohort of 207 pairs of jumps and demonstrated the adjustments made by athletes to achieve bar clearance after unsuccessful jumps. Results revealed that all groups showed a significant, despite small in magnitude, increase in take-off speed at the successful compared to the unsuccessful jumps. In addition, only small differences were observed between the successful and unsuccessful jumps for the rest of the examined parameters, which suggest that, for trained pole vaulters, small changes in the kinematics of the approach phase can influence the outcome of the jump.

Speed at last touchdown was slightly larger for all groups in the successful compared to unsuccessful jumps. Similarly, average approach speed at the $10-5 \mathrm{~m}$ section was greater in the successful jumps for all groups except for elite women. This finding is in agreement with previous research that suggests that speed is the major determinant of performance in pole vault for men and women (Adamczewski \& Perlt, 1997; Cassirame, Sanchez, Homo, \& Frère, 2017; Linthorne \& Weetman, 2012; McGinnis, 2004). The small increase in speed observed in the successful jumps is beneficial, as it is suggested that larger speed at take-off can lead to a higher initial energy that an athlete could transmit to the pole, which in turn increases the flexion of the pole and enhances the recoil energy return (Linthorne \& Weetman, 2012; Schade, Arampatzis, \& Bruggemann, 2000).

Progression of approach speed was assessed by measuring speed difference between each 5-m section of the approach run. In this study, findings showed that athletes increased their speed throughout the approach run as found in past research (Linthorne \& Weetman, 2012). Small differences were observed for the progression of speed from the 10-5 $\mathrm{m}$ section to the last touchdown between successful and unsuccessful jumps in all groups, except the cadet women. Average approach speed at the $15-10 \mathrm{~m}$ section was also higher (with small effect) in successful jumps for cadet women and junior males, while average approach speed at the 20$15 \mathrm{~m}$ section was larger at the successful jumps only for the cadet women. The above finding provides an additional argument relating higher speed at the end of the approach run with better pole vault performance.

It was also noted that few approach step parameters were different between successful and unsuccessful jumps. A small decrease of aerial time was reported for junior men and women in the successful compared to the unsuccessful jumps. Additionally, a small increase of step rate for junior women and of step length for cadet women was observed in the successful compared to the unsuccessful jumps. The adjustments in these parameters allow athletes to increase running speed as been reported in several previous studies investigating running performance (Chapman \& Caldwell, 1983; Miller, Umberger, \& Caldwell, 2012; Rabita et al., 2015). Nevertheless, pole carriage impairs the development of maximum speed due to the decrement in stride length because of the reduced maximal hip and knee flexion (Frère, 
Chollet, \& Tourny-Chollet, 2009). Furthermore, carrying a pole was found to alter both the horizontal force and velocity capabilities of an athlete, a combination that has an effect on the horizontal power production (Frère et al., 2017). These factors are the bases of gender differences in sprinting in adolescents (Papaiakovou et al., 2009) and might explain the modification of step parameters in cadet and junior women. However, the increased reliance on step rate during the approach has previously been highlighted for the achievement of a successful compared to an unsuccessful jump (Theodorou, Panoutsakopoulos, Exell, \&

\section{Vujkov, 2017).}

243

As mentioned previously, cadet women did not differentiate the progression of approach speed between successful and unsuccessful jumps at the very last part of the approach. Contrary to long jump or triple jump, pole vault includes an important impact during take-off where the athlete has to transmit energy to the pole instead of absorbing the energy (Christensen et al., 2014; Plessa, Rousanoglou, \& Boudolos, 2010; Schade, Arampatzis, \& Brüggemann, 2006). This phase is probably the most crucial moment in pole vaulting since a number of musculo-skeletal injuries occur (Rebella, 2015). This greater stress on take-off is suggested to force athletes unconsciously to reduce their velocity prior to planting the pole to protect their body (Goligorsky, 2001) and to act in a preventing status, with less variability during the approach (Hay, 1988). The above observations about sprinting parameters may indicate that fine adjustments of these variables between successful and unsuccessful jumps may lead to changes in step rate and length and hence take-off speed. Previous research suggested that differences exist in gait regulation strategy between successful and unsuccessful jumps, with less step placement variability at the final steps of the approach (Tamura et al., 2017). This is not supported by the present study,as no differences were observed for step length asymmetry and variability or step length adjustment. Theodorou et al. (2016) also noted the absence of step parameters asymmetry in late approach of elite male pole vaulters. This might be due to the higher level of athletes examined in the present study, as athletes of a higher skill level were found to exhibit a variety of motor response patterns and greater success rates (Needham, Bezodis, Exell, \& Irwin, 2017).

Regarding the take-off posture, successful jumps were performed with greater negative values for the horizontal grip hand and take-off foot's toes distance at both pole plant and take-off except for cadet and elite males. In addition, a more proximal position to the planting box was noted for junior women, elite women and junior male. These findings are not in agreements with the traditional Russian pole vault technique, where a more distal take-off position is favored combined with positive or close to zero values regarding the horizontal grip hand and take-off foot's toes distance at both pole plant and take-off (Vaslin \& Cid, 1993). Results showed that, in the successful jumps, the grip hand was more posterior in relation to the takeoff foot at heel strike and toe-off. This position probably allowed athletes to obtain better active energy transmission to the pole during floor contact and initiated pole bending (McGinnis, 1997; Schade \& Arampatzis, 2012). As described by Warburton et al. (2015), the largest force required to bend the pole occurs at the first part of the bending. Furthermore, a closer take-off distance, in combination with greater horizontal grip hand and take-off foot's toes distance at both pole plant and take-off, allows an increase in the duration of pole bending when the foot is on the floor and thus permitting a better force transmission to the 
pole. In addition, the increased speed at the end of the approach could allow better energy transfer between the athlete and the pole, increasing momentum at take-off.

Traditional approaches to pole vault suggest that longer take-off distance (Gudelj et al., 2015), higher grip length (Sullivan, Knowlton, Hetzler, \& Woelke, 1994), larger pole to floor angle and the use of a stiffer pole can also lead to improved performance (Linthorne, 2000). With this technical approach, vaulters need to use their own energy to straighten the pole when clearing the bar. However, the results of this study suggest that vaulters may require a different technical approach in response to improved pole proprieties (Ekevad \& Lundberg, 1997; Schade \& Arampatzis, 2012), including a closer take-off position and larger foot-hand separation parameters. This observation is corroborated by the height of grip hand at take-off for elite male, demonstrating a lower position of the grip hand in the successful compared to the unsuccessful jumps. In the successful jumps, athletes appear to attempt to produce maximal pole bending during the last ground contact instead of preparing for pole straightening. For each athlete's grip length and height, it is suggested that they have an ideal take-off position to maximize horizontal speed produced during the run-up and subsequent pole bending (Linthorne, 2000). The findings of this study suggest that athletes maximized foot-hand separation positions in order to succeed when vaulting.

Due to the use or real-world competition data, this study compared successful and unsuccessful jump attempts in this order. It is unclear whether the changes between conditions resulted from negative changes to technique in the unsuccessful jumps or positive changes to technique leading to successful jumps. Changes in values between successful and unsuccessful jumps may have been induced by the change in position of athletes' start mark for the first attempt at a new bar height. Under this condition, the athlete could wrongly adjust their take-off position and perform an unsuccessful jump. The second or third attempts however may allow them to modify this position in response to the failed attempt to obtain a positive result (Theodorou, Panoutsakopoulos, Exell, \& Vujkov, 2017). In addition, pole vault is a highly complex discipline with a large number of potential causes for unsuccessful jumps. This study has used a large data set covering a range of ability levels to initially describe differences in technique between unsuccessful and successful jumps, but has only considered run-up parameters and take-off position. Therefore, future work should analyze the flight phase of the jump for the bar clearance for further information concerning the technique elements that distinguish unsuccessful and successful pole vault jumps.

\section{Practical application}

The results from this study can inform coaches and athletes to focus on the most relevant points to producing successful jumps. During competition, these findings highlight the importance to reach maximum speed capability during the approach run. In addition, reducing hand-foot distance at take-off can also increase the possibility of a successful jump by increasing initial pole bending when the athlete is in contact with the floor. It was noted that the elite male analysed in this study broke down the traditional Russian technique by focusing on a bigger pole-to-floor angle at take-off aiming for a quicker loading of the pole. Further investigation into this phenomenon would be beneficial to confirm whether greater horizontal take-off orientation can maximize energy transfer and jump results. 


\section{Conclusion}

323 This present study compared successful and unsuccessful jumps in pole vault. The main

324 finding of this study is that successful jumps were associated with a faster approach run and a

325 faster horizontal speed at the take-off phase. This suggests that athletes should try to produce

326 as high velocity as possible during the approach run to improve the likelihood of successful

327 jumps, as small reductions in speed can lead to failed attempts. The findings also suggest that

328 athletes can modify the horizontal distance of the toe of the take-off foot from the end of the

329 plant box at take-off and the horizontal displacement of the superior hand from the take-off

330 foot's toes at pole plant and the instant of take-off in order to achieve a successful jump. The

331 findings of the present study highlight the complexity of the pole vault task and that he small

332 margins between successful and unsuccessful jumps and the potential detrimental effect of the

333 variability of the kinematical parameters for a successful jump. Future studies should focus on

334 obtaining better understanding of mechanisms responsible for improving this energy transfer

335 and analysis of athletes' technique during the bar clearance.

\section{Acknowledgment}

338 The authors declare that there was no conflict of interest with the results presented in this 339 study. The authors would like to thank the French Federation of Athletics for the approval and 340 support during data acquisition at the National Championships. Appreciation extended to Mr. 341 Renaud Lavillenie, the organizer of the All-Star Perche meeting. This study was conducted

342 with material support by the Matsport Training Company and the EPSI platform from 343 Besançon. In addition, the authors thank Mr. Simon Chevrolat for his permition to use the 344 MookyPVcompiler software that assisted to the fast computation of the data. 
References

Adamczewski, H., \& Perlt, B. (1997). Run-up velocities of female and male pole vaulting and some technical aspects of women's pole vault. New Studies in Athletics, 12(1), 63-76.

Ammann, R., Taube, W., \& Wyss, T. (2016). Accuracy of PARTwear inertial sensor and Optojump optical measurement system for measuring ground contact time during running. Journal of Strength and Conditioning Research, 30(7), 2057-2063. doi: 10.1519/JSC.0000000000001299

Angulo-Kinzler, R. M., Kinzler, S. B., Balius, X., Turro, C., Caubet, J. M., Escoda, J., \& Prat, J. A. (1994). Biomechanical analysis of the pole vault event. Journal of Applied Biomechanics, 10(2), 147-165. doi: 10.1123/jab.10.2.147

Cassirame, J., Sanchez, H., Homo, S., \& Frère, J. (2017). Mechanical performance determinants in women's vs men's pole-vault. Computer Methods in Biomechanics and Biomedical Engineering, 20(Suppl. 1), 37-38. doi: 10.1080/10255842.2017.1382849

Cassirame, J., Sanchez, H., \& Morin, J. B. (2018). The elevated track in pole vault: An advantage during run-up? International Journal of Sports Physiology and Performance, 13(6), 717-723. doi:10.1123/ijspp.2016-0724

Chapman, A. E., \& Caldwell, G. E. (1983). Kinetic limitations of maximal sprinting speed. Journal of Biomechanics, 16(1), 79-83. doi: 10.1016/0021-9290(83)90048-9

Christensen, B. (2004). Individual biomechanical profiles of changes in technique between practice and competition in seven collegiate pole vaulters. In: M. Lamontagne, D. G. E. Robertson, \& H. Sveistrup (Eds), Proceedings of the $22^{\text {nd }}$ International Conference of Biomechanics in Sports (pp. 527-530). Ottawa: I.S.B.S.

Christensen, B., Francis, S., Keller, S., Strand, B., \& Hatterman-Valenti, H. (2014). An examination of ground reaction forces of three pole vault take-off styles. In: K. Sato, W. A. Sands, \& S. Mizuguchi (Eds), Proceedings of the $32^{\text {nd }}$ International Conference of Biomechanics in Sports (pp. 520-522). Johnson City, TN: I.S.B.S.

Christensen, B., \& Zebas, C. (2000). A comparison of practice and competition approach velocities and the position of the top handhold at the pole plant in pole vaulters. In: Y. Hong, D. P. Johns, \& R. Sanders (Eds): Proceedings of the $18^{\text {th }}$ International Conference of Biomechanics in Sports. Hong Kong: I.S.B.S.

Decker, A., \& Bird, M. (2004). Predicting potential jump height in the pole vault from four variables. In: M. Lamontagne, D. G. E. Robertson, \& H. Sveistrup (Eds), Proceedings of the $22^{\text {nd }}$ International Conference of Biomechanics in Sports (pp. 250-252). Ottawa: I.S.B.S.

Ekevad, M., \& Lundberg, B. (1997). Influence of pole length and stiffness on the energy conversion in pole-vaulting. Journal of Biomechanics, 30(3), 259-264. doi: 10.1016/S0021-9290(96)00131-5

Frère, J., Chollet, D., \& Tourny-Chollet, C. (2009). Assessment of the influence of pole carriage on sprint kinematics: A case study of novice athletes. International Journal of Sports Science and Engineering, 3(1), 3-10 
400

401

402

403

404

405

406

407

408

409

410

411

412

413

414

415

416

417

418

419

420

421

422

423

424

425

426

427

428

429

430

431

432

Frère, J., L'hermette, M., Slawinski, J., \& Tourny-Chollet, C. (2010). Mechanics of pole vaulting: a review. Sports Biomechanics, 9(2), 123-138. doi: 10.1080/14763141.2010.492430.

Frère, J., Sanchez, H., Homo, S., Rabita, G., Morin, J. B., \& Cassirame, J. (2017). Influence of pole carriage on sprint mechanical properties during pole vault run-up. Computer Methods in Biomechanics and Biomedical Engineering, 20(Suppl. 1), 83-84. doi: 10.1080/10255842.2017.1382872

Gudelj, I., Babić, V., Milat, S., Čavala, M., Zagorac, S., \& Katić, R. (2015). Differences in some kinematic parameters between two qualitatively different groups of pole vaulters. Collegium Antropologicum, 39(Suppl. 1), 41-46.

Goligorsky, M. S. (2001). The concept of cellular "fight-or-flight" reaction to stress. American Journal of Physiology-Renal Physiology, 280(4), F551-F561. doi: 10.1152/ajprenal.2001.280.4.F551

Hay, J. G. (1988). The approach run in the pole vault. Track Technique, 106, 3376-3378.

Hopkins, W., Marshall, S., Batterham, A., \& Hanin, J. (2009). Progressive statistics for studies in sports medicine and exercise science. Medicine and Science in Sports and Exercise, 41(1), 3-13. doi: 10.1249/MSS.0b013e31818cb278

Linthorne, N. P. (2000). Energy loss in the pole vault take-off and the advantage of the flexible pole. Sports Engineering, 3(4), 205-218. doi: 10.1046/j.14602687.2000.00058.x

Linthorne, N. P., \& Weetman, A. G. (2012). Effects of run-up velocity on performance, kinematics, and energy exchanges in the pole vault. Journal of Sports Science and Medicine, 11(2), 245-254.

Makaruk, H., Porter, M., Starzak, M., \& Szymczak, E. (2016). An examination of approach run kinematics in track and field jumping events. Polish Journal of Sport and Tourism, 23(2), 82-87. doi: 10.1515/pjst-2016-0009

McGinnis, P. (1997). Approach run velocities of female pole vaulters. In: J. Wilkerson, K. Ludwig, \& W. Zimmermann (Eds), Proceedings of the $15^{\text {th }}$ International Conference of Biomechanics in Sports (pp. 101-105). Denton, TX: I.S.B.S.

McGinnis, P. M. (2004). Evolution of the relationship between performance and approach run velocity in the women's pole vault. In: M. Lamontagne, D. G. E. Robertson, \& H. Sveistrup (Eds), Proceedings of the $22^{\text {nd }}$ International Conference of Biomechanics in Sports (pp. 531-534). Ottawa: I.S.B.S.

Miller, R. H., Umberger, B. R., \& Caldwell, G. E. (2012). Limitations to maximum sprinting speed imposed by muscle mechanical properties. Journal of Biomechanics, 45(6), 1092-1097. doi: 10.1016/j.jbiomech.2011.04.040

Needham, L., Bezodis, I., Exell, T., \& Irwin, G. (2017). Pole-athlete interaction during the pole vault approach phase. In: Proceedings of the $35^{\text {th }}$ International Conference of Biomechanics in Sports (pp. 781-784). Cologne: I.S.B.S.

Needham, L., Exell, T. A., Bezodis, I. N., \& Irwin, G. (2018). Patterns of locomotor regulation during the pole vault approach phase. Journal of Sports Sciences, 36(15), 1742-1748. doi: 10.1080/02640414.2017.1412236

Papaiakovou, G., Giannakos, A., Michailidis, C., Patikas, D., Bassa, E., Kalopisis, V., ... \& Kotzamanidis, C. (2009). The effect of chronological age and gender on the 
development of sprint performance during childhood and puberty. Journal of Strength and Conditioning Research, 23(9), 2568-2573. doi: 10.1519/JSC.0b013e3181c0d8ec

Plessa, E. I., Rousanoglou, E. N., \& Boudolos, K. D. (2010). Comparison of the take-off ground reaction force patterns of the pole vault and the long jump. Journal of Sports Medicine and Physical Fitness, 50(4), 416-421.

Rabita, G., Dorel, S., Slawinski, J., Sàez-de-Villarreal, E., Couturier, A., Samozino, P., \& Morin, J. B. (2015). Sprint mechanics in world-class athletes: a new insight into the limits of human locomotion. Scandinavian Journal of Medicine and Science in Sports, 25(5), 583-594. doi: 10.1111/sms. 12389

Rebella, G. (2015). A prospective study of injury patterns in collegiate pole vaulters. American Journal of Sports Medicine, 43(4), 808-815. doi: $10.1177 / 0363546514564542$

Schade, F., \& Arampatzis, A. (2012). Influence of pole plant time on the performance of a special jump and plant exercise in the pole vault. Journal of Biomechanics, 45(9), 1625-1631. doi: 10.1016/j.jbiomech.2012.03.031

Schade, F., Arampatzis, A., \& Brüggemann, G. P. (2006). Reproducibility of energy parameters in the pole vault. Journal of Biomechanics, 39(8), 1464-1471. doi: 10.1016/j.jbiomech.2005.03.027

Schade, F., Arampatzis, A., \& Brüggemann, G. P. (2000). Influence of different approaches for calculating the athlete's mechanical energy on energetic parameters in the pole vault. Journal of Biomechanics, 33(10), 1263-1268. doi: 10.1016/S00219290(00)00087-7

Schade, F., Arampatzis, A., Brüggemann, G. P., \& Komi, P. V. (2004). Comparison of the men's and the women's pole vault at the 2000 Sydney Olympic Games. Journal of Sports Sciences, 22(9), 835-842. doi: 10.1080/02640410410001675315

Starzak, M., Makaruk, H., \& Niznikowski, T. (2016). The effects of different task constraints on variability of footfall placement in novice pole vaulters. In: K. J. Slomka \& G. Juras (Eds), Current Research in Motor Control V (pp. 300-305). AWF: Katowice.

Sullivan, J. J., Knowlton, R. G., Hetzler, R. K., \& Woelke, P. L. (1994). Anthropometric characteristics and performance related predictors of success in adolescent pole vaulters.Journal of Sports Medicine and Physical Fitness, 34(2), 179-184.

Tamura, Y., Nunome, H., \& Usui, S. (2017). The Difference in Gait Regulation Strategies Between Successful and Failed Pole Vault Performance. Journal of Sports Science 5, 4, 211-214. doi: 10.17265/2332-7839/2017.04.004

Theodorou, A., Panoutsakopoulos, V., \& Exell, T. (2016). Step characteristic interaction and asymmetry during the approach phase in pole vault. Journal of Sports Sciences, 34(Suppl. 1), s43.

Theodorou, A., Panoutsakopoulos, V., Exell, T., \& Vujkov, N. (2017). Comparison of step characteristic interaction and asymmetry between failed and succesful attempts in pole vault. In: Proceedings of the 35th Conference of the International Society of Biomechanics in Sports (pp. 777-780). Cologne: I.S.B.S.

Theodorou, A. S., Panoutsakopoulos, V., Exell, T. A., Argeitaki, P., Paradisis, G. P., \& Smirniotou, A. (2017). Step characteristic interaction and asymmetry during the approach phase in long jump. Journal of Sports Sciences, 35(4), 346-354. doi: $10.1080 / 02640414.2016 .1164884$ 
Vaslin, P., \& Cid, M. (1993). Les facteurs de la performance en saut à la perche dans la littérature scientifique. STAPS-Sciences et Techniques des Activités Physiques et Sportives, 31, 75-86.

Warburton, T., James, R., Lyttle, A., \& Alderson, J. (2016). An analysis of different pole vaulting pole load-deformation testing regimes. In: F. Colloud, M. Domalain, \& T. Monnet (Eds), Proceedings of the $33^{\text {rd }}$ Conference of the International Society of Biomechanics in Sports (pp. 745-748). Poitiers: I.S.B.S.

Zagorac, N. (2013). Influence of kinematic parameters on pole vault result in top junior athletes. Collegium Antropologicum, 37(Suppl. 2), 19-24. 
Figure 1. Experimental set-up illustration including the Optojump Next system and the radar gun.

Figure 2. Schematic representation of the video-based measurements in Position 1 (pole plant) and Position 2 (take-off).

Figure 3. Difference between successful and unsuccessful jumps for cadet (CW), junior (JW) and elite (EW) women for each parameter. Differences are expressed in SD. The grey area represents no significant differences between both conditions; * denotes a small difference.

Figure 4. Difference between successful and unsuccessful jumps for cadet (CM), junior (JM) and elite (EM) men for each parameter. Differences are expressed in SD. The grey area represents no significant differences between both conditions; * denotes a small difference.

Table 1. Mean $\pm S D$ results for the step parameters for the successful and unsuccessful jumps. Parameters highlighted in grey are significantly different between both conditions.

NOTE: * denotes a small difference. For each category, the number of jump duos analyzed for comparison is 36 for Cadet Women, 29 for Junior Women, 38 for Elite Women, 36 for Cadet Men, 35 for Junior Men and 39 for Elite Men.

PoTk: distance from planting box at take-off, Po6S: distance from box at the $6^{\text {th }}$-to-last step, SL: step length, SR: step rate, $\mathrm{SL}_{\text {asy }}$ : step length asymmetry, $\mathrm{SL}_{\mathrm{var}}$ : step length variability, SLadj: step length adjustment: $t_{a}$ : aerial time, $t_{c}$ : contact time.

Table 2. Mean $\pm S D$ results for the speed parameters for the successful and unsuccessful jumps. Parameters highlighted in grey are significantly different between both conditions.

NOTE: * denotes a small difference. For each category, the number of jump duos analyzed for comparison is 36 for Cadet Women, 29 for Junior Women, 38 for Elite Women, 36 for Cadet Men, 35 for Junior Men and 39 for Elite Men.

Sp1: average approach speed at the $20-15 \mathrm{~m}$ section, Sp2: average approach speed at the 15$10 \mathrm{~m}$ section, Sp3: average approach speed at the 10-5 m section, SpTK: speed at last touchdown, $\Delta 1$ : progression of speed between $\mathrm{Sp} 1$ and $\mathrm{Sp} 2, \Delta 2$ : progression of speed between $\mathrm{Sp} 2$ and Sp3, $\Delta 3$ : progression of speed between Sp3 and SpTK.

Table 3. Mean $\pm S D$ results for the 2D kinematical analysis parameters for the successful and unsuccessful jumps. Parameters highlighted in grey are significantly different between both conditions.

NOTE: * denotes a small difference. For each category, the number of jump duos analyzed for comparison is 36 for Cadet Women, 29 for Junior Women, 38 for Elite Women, 36 for Cadet Men, 35 for Junior Men and 39 for Elite Men.

$\mathrm{H} 1$ : height of grip/upper hand at pole plant, $\mathrm{H} 2$ : height of grip/upper hand at take-off, $\Delta \mathrm{H}$ : $\mathrm{H} 2-\mathrm{H} 1, \mathrm{U} 1$ : horizontal distance between grip hand and take-off foot's toes at pole plant, U2: horizontal distance between grip hand and take-off foot's toes at take-off, $\Delta \mathrm{U}$ : U2-U1. 
Table 1. Average $\pm S D$ results from step parameters measurement sessions for successful and failed attempts. Parameters highlighted in grey are significantly different between both conditions.

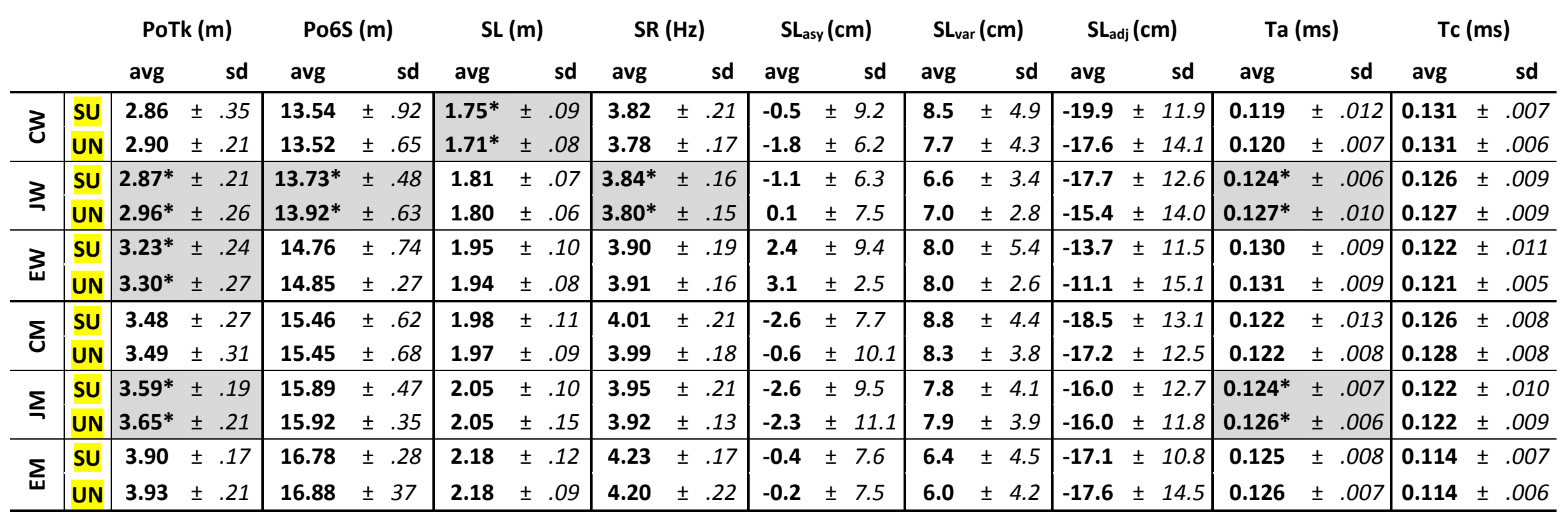

NOTE: * denotes a small difference. For each category numbers of jump duos analyzed for comparison are 36 for CW, 29 for JW, 38 for EW, 36 for CM, 35 for JM and 39 for EM.

PoTk: Position as take-off, Po6S: position at 6 strides, SL: stride length, SR: stride rate, SLasy: stride length asymmetry, SLvar: stride length variability SLadj: stride length adjustment: ta: aerial time, tc : contact time 
Table 2. Average $\pm S D$ results from speed parameters measurement sessions for successful and failed attempts. Parameters highlighted in grey are significantly different between both conditions.

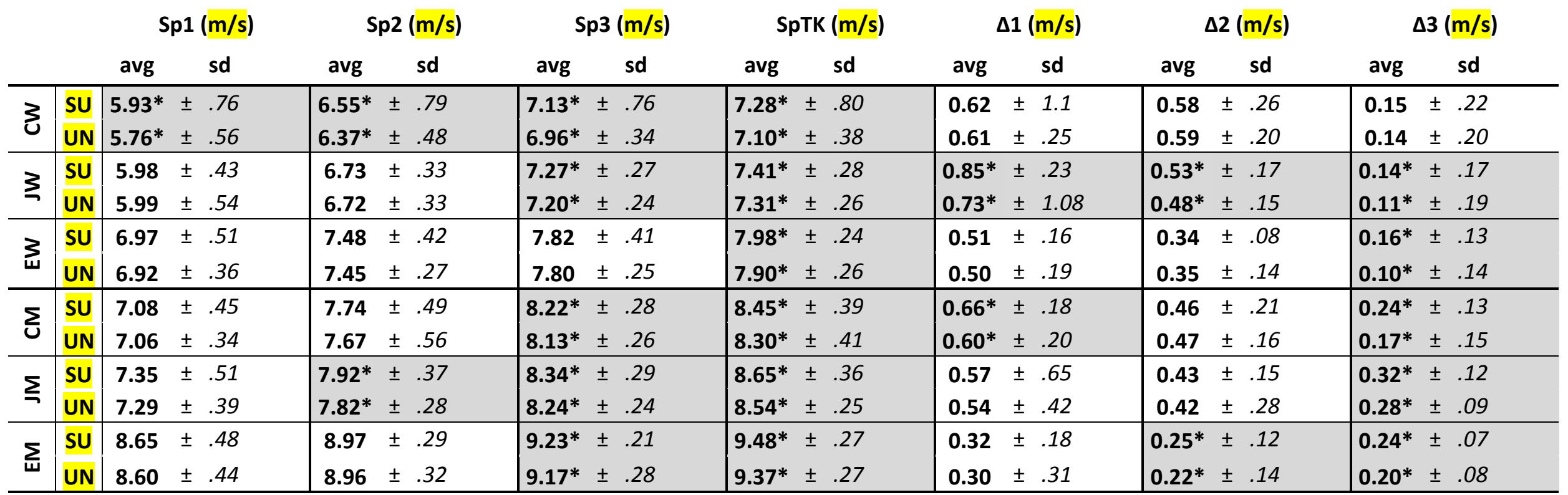

NOTE: * denotes a small difference. For each category numbers of jump duos analyzed for comparison are 36 for CW, 29 for JW, 38 for EW, 36 for CM, 35 for JM and 39 for EM.

SP1: avg speed 20-15m, SP2: avg speed 15-10m, SP3 avg speed 10-5 m, SPTk: speed at take-off, $\Delta 1:$ speed evolution between Sp1 and SP2, $\Delta 2$ : Speed evolution between SP2 and SP3, 43 , Speed evolution between SP3 and SPTk, 
Table 3. Average $\pm S D$ results from $2 \mathrm{D}$ kinematical analysis parameters measurement sessions for successful and failed attempts. Parameters highlighted in grey are significantly different between both conditions.

\begin{tabular}{|c|c|c|c|c|c|c|c|c|c|c|c|c|c|c|c|c|c|c|c|}
\hline & & \multicolumn{3}{|c|}{$\mathrm{H} 1(\mathrm{~cm})$} & \multicolumn{3}{|c|}{$\mathrm{H} 2(\mathrm{~cm})$} & \multicolumn{3}{|c|}{$\Delta H(\mathrm{~cm})$} & \multicolumn{3}{|c|}{$\mathrm{U} 1(\mathrm{~cm})$} & \multicolumn{3}{|c|}{$\mathrm{U} 2(\mathrm{~cm})$} & \multicolumn{3}{|c|}{$\Delta U(\mathrm{~cm})$} \\
\hline & & avg & & sd & avg & & $s d$ & avg & & sd & avg & & sd & avg & & sd & avg & & sd \\
\hline \multirow{2}{*}{3} & SU & 183.7 & \pm & 9.0 & 193.2 & \pm & 6.5 & 9.5 & \pm & 5.8 & $-36.9 *$ & \pm & 20.4 & $-18.5^{*}$ & \pm & 16.3 & 18.4 & \pm & 9.9 \\
\hline & UN & 182.6 & \pm & 9.5 & 193.0 & \pm & 10.9 & 10.4 & \pm & 6.1 & $-30.8 *$ & \pm & 19.5 & $-13.3^{*}$ & \pm & 17.2 & 17.4 & \pm & 11.7 \\
\hline \multirow{2}{*}{3} & SU & 180.7 & \pm & 10.4 & 189.1 & \pm & 11.6 & 8.3 & \pm & 3.7 & $-37.7 *$ & \pm & 14.2 & $-17.8^{*}$ & \pm & 11.9 & $20.0 *$ & \pm & 9.6 \\
\hline & UN & 181.9 & \pm & 12.5 & 189.6 & \pm & 12.8 & 7.6 & \pm & 6.7 & $-30.9 *$ & \pm & 21.1 & $-13.4^{*}$ & \pm & 18.2 & $17.4^{*}$ & \pm & 9.1 \\
\hline \multirow{2}{*}{3} & SU & 191.8 & \pm & 10.1 & 200.5 & \pm & 10.3 & 8.7 & \pm & 5.3 & $-32.9 *$ & \pm & 10.2 & $-14.9 *$ & \pm & 8.1 & 18.0 & \pm & 12.0 \\
\hline & UN & 190.8 & \pm & 6.0 & 200.5 & \pm & 6.2 & 9.6 & \pm & 4.7 & $-27.6 *$ & \pm & 8.7 & $-10.8^{*}$ & \pm & 6.4 & 16.8 & \pm & 9.7 \\
\hline \multirow{2}{*}{$\sum_{\bigcup}$} & SU & 193.8 & \pm & 12.3 & 204.2 & \pm & 9.9 & 10.4 & \pm & 3.2 & -33.4 & 1 & 15.0 & -12.7 & \pm & 7.5 & 20.1 & \pm & 7.8 \\
\hline & UN & 194.1 & \pm & 11.4 & 204.5 & \pm & 8.9 & 10.4 & \pm & 4.5 & -31.4 & \pm & 12.5 & -12.4 & \pm & 6.4 & 18.5 & \pm & 12.8 \\
\hline \multirow{2}{*}{$\sum$} & SU & 198.9 & \pm & 13.2 & 207.2 & \pm & 10.5 & 8.3 & \pm & 6.5 & $-16.4 *$ & \pm & 8.5 & $-34.2 *$ & \pm & 11.4 & 17.9 & \pm & 10.4 \\
\hline & UN & 199.1 & \pm & 11.2 & 207.9 & \pm & 10.6 & 8.7 & \pm & 4.2 & $-11.6 *$ & \pm & 7.4 & $-27.5^{*}$ & \pm & 10.4 & 16.0 & \pm & 12.5 \\
\hline \multirow{2}{*}{$\sum_{\mathbf{W}}$} & SU & 207.3 & \pm & 7.5 & 207.0* & \pm & 7.4 & $-0.3^{*}$ & \pm & 4.2 & -43.6 & 1 & 15.3 & -21.1 & \pm & 13.4 & 22.4 & \pm & 9.7 \\
\hline & UN & 207.3 & \pm & 6.4 & 213.3* & \pm & 6.1 & $6.0^{*}$ & \pm & 5.2 & -43.3 & \pm & 11.4 & -21.7 & \pm & 11.2 & 21.5 & \pm & 9.5 \\
\hline
\end{tabular}

NOTE: * denotes a small difference. For each category numbers of jump duos analyzed for comparison are 36 for CW, 29 for JW, 38 for EW, 36 for CM, 35 for JM and 39 for EM.

$\mathrm{H} 1$ and $\mathrm{H} 2$ : height of upper hand at position 1 and 2, $\Delta \mathrm{H}: \mathrm{H} 2-\mathrm{H} 1, \mathrm{U} 1$ and $\mathrm{U} 2$ : under values at position 1 and 2, $\Delta \mathrm{U}: \mathrm{U} 2-\mathrm{U}$ 


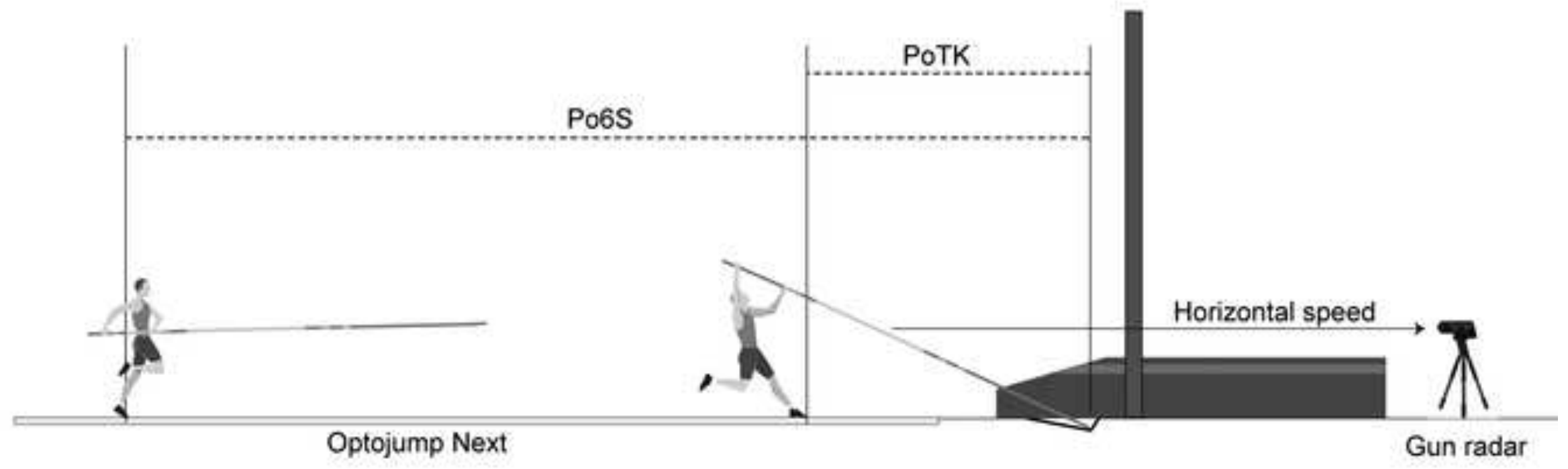




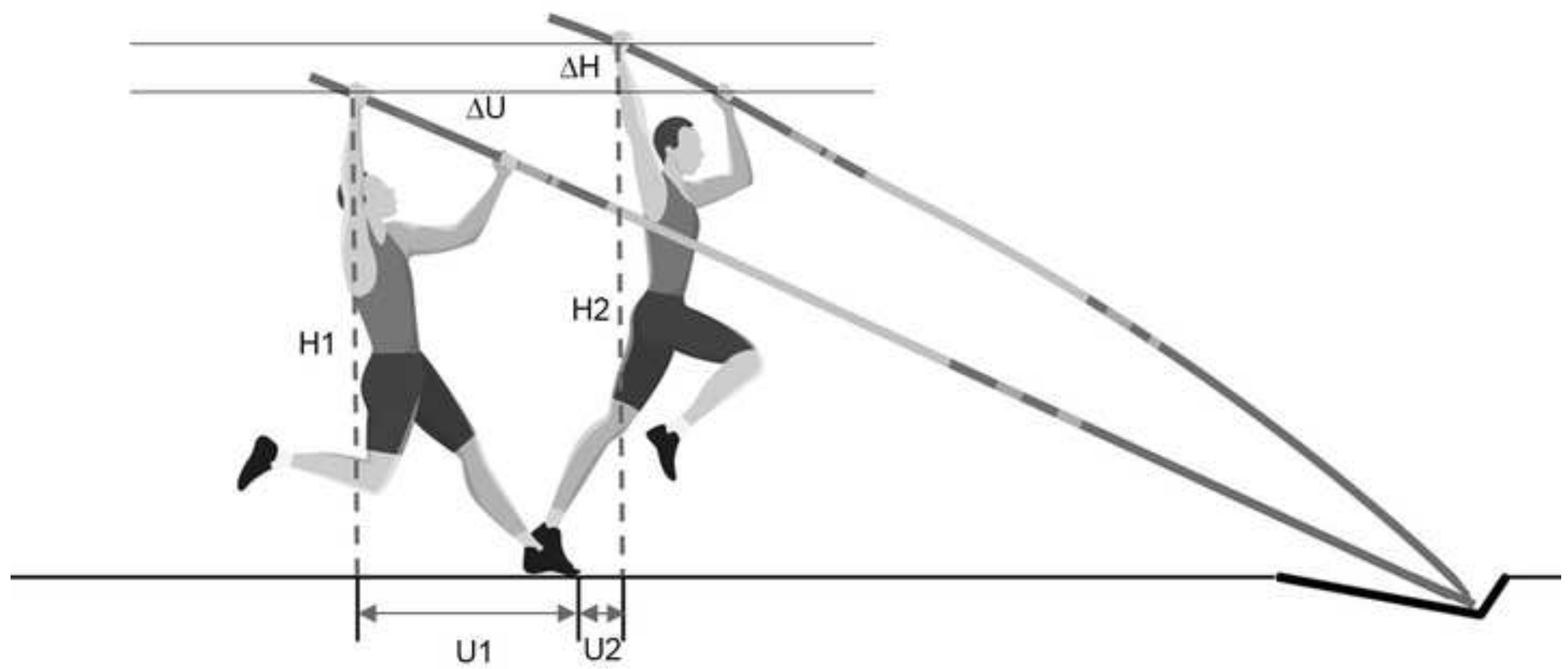


Cadet Women Junior Women Elite Women

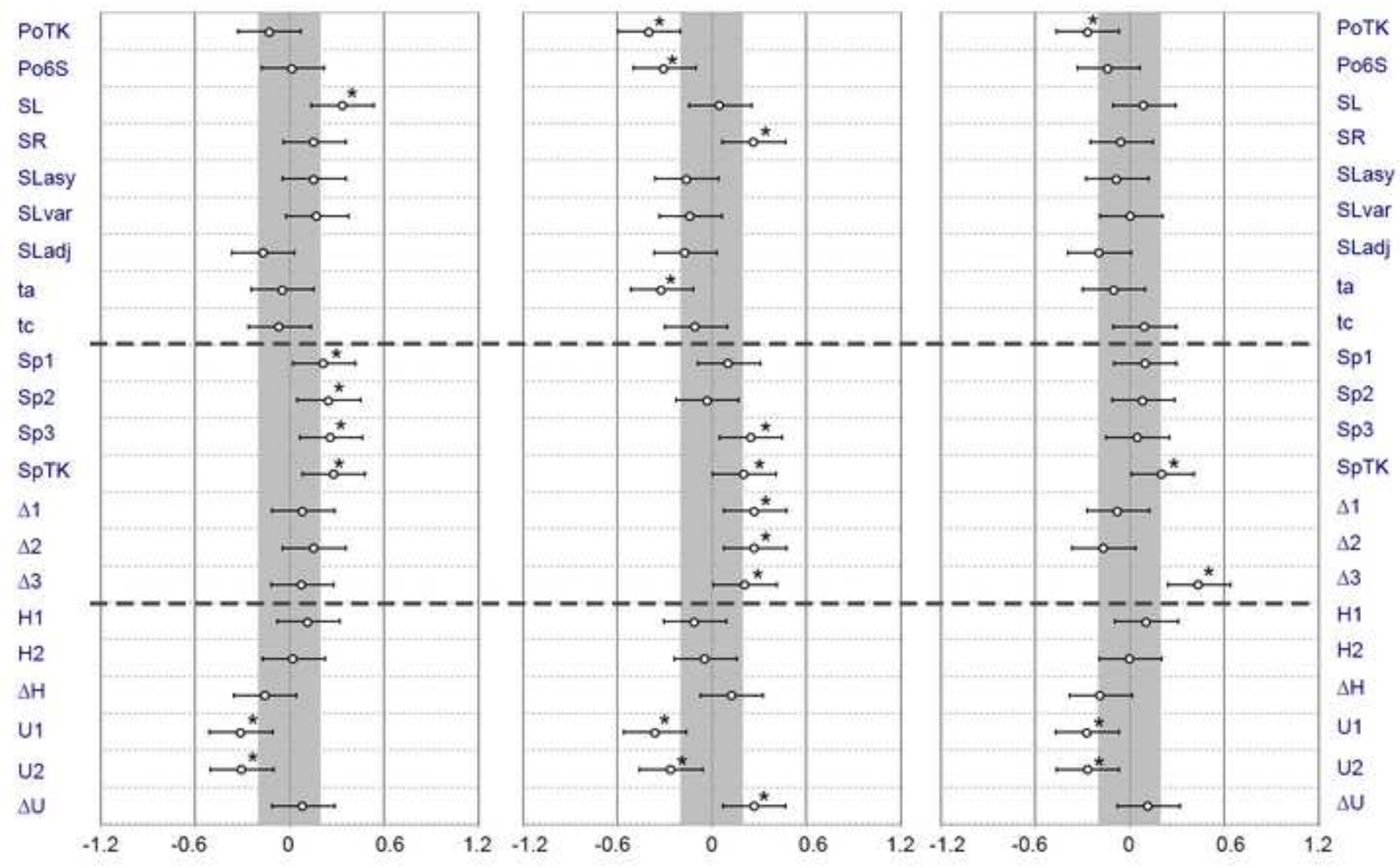




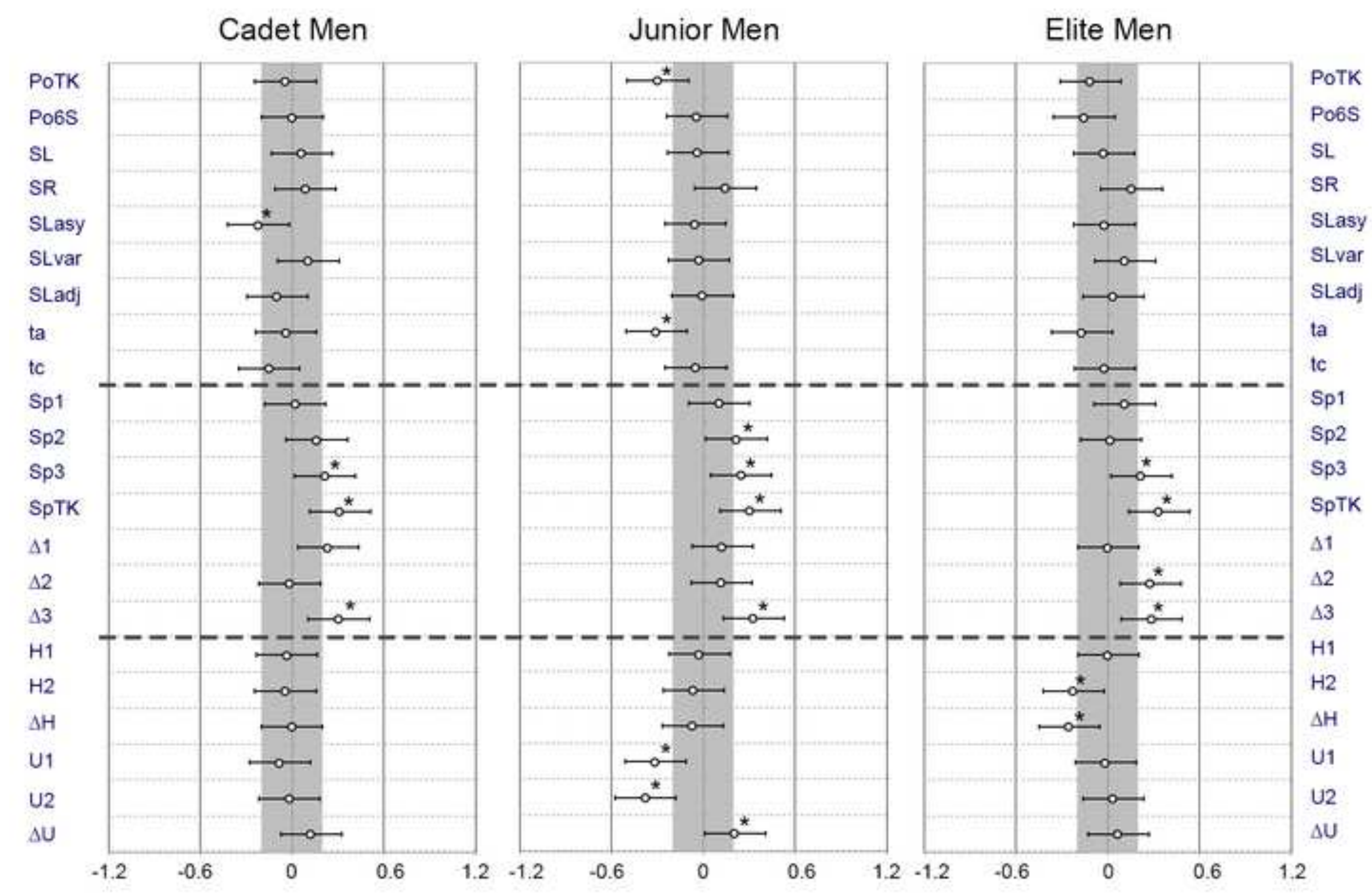

